\title{
TRAUMATIC BRAIN INJURY-ASSOCIATED MICRGOGLIA ADOPT LONGITUDINAL TRANSCRIPTIONAL CHANGES CONSISTENT WITH LONG-TERM DEPRESSION OF SYNAPTIC STRENGTH
}

Hadijat M. Makinde ${ }^{1}$, Talia B. Just ${ }^{1}$, Deborah R. Winter ${ }^{2 \pi}$, Steven J. Schwulst ${ }^{1 * \pi{ }^{*}}$

${ }^{1}$ Department of Surgery, Division of Trauma and Critical Care, Northwestern University, Chicago, Illinois, United States of America

2 Department of Medicine, Division of Rheumatology, Northwestern University, Chicago, Illinois, United States of America

${ }^{*}$ Corresponding author

E-Mail: s-schwulst@northwestern.edu

ISJS and DP are Joint Senior Authors 
1 Traumatic brain injury ( $\mathrm{TBI})$ is an under-recognized public health threat. Even mild brain

2 injury, or concussions, may lead to long-term neurologic impairment. Microglia play a

3 fundamental role in the development and progression of this subsequent neurologic

4 impairment. Despite this, a microglia-specific injury signature has yet to be identified. In

5 the current study we hypothesized that TBI-associated microglia would adopt longitudinal

6 changes in their transcriptional profile associated with pathways linked to the

7 development of motor, cognitive, and behavioral disorders. C57BL/6 mice underwent TBI

8 via a controlled cortical impact and were followed longitudinally. FACSorted microglia

9 from TBI mice were subjected to RNA-sequencing at 7, 30, and 90 days post-injury. We

10 identified 4 major patterns of gene expression corresponding to the host defense

11 response, synaptic potentiation, lipid remodeling, and membrane polarization. In

12 particular, significant upregulation of genes involved in long-term synaptic potentiation

13 including Ptpn5, Shank3, and Sqstm1 were observed offering new insight into a

14 previously unknown role of microglia in the weakening of synaptic efficacy between

15 neurons after brain injury.

\section{Introduction}

18 Traumatic brain injury is a growing and under recognized public health threat. The CDC

19 estimates nearly 2 million people sustain a traumatic brain injury (TBI) each year in the

20 United States, contributing to over $30 \%$ of all injury related deaths ${ }^{1,2}$. In fact, TBI related

21 healthcare expenditures near 80 billion dollars annually with an average cost of 4 million

22 dollars per person surviving a severe $\mathrm{TBI}^{3-5}$. The impact of $\mathrm{TBI}$ is highlighted not only by

23 its high mortality rate but also by the significant long-term complications suffered by its 
24 survivors with the progressive development of motor, cognitive, and behavioral disorders

$25 \quad 6-10$. Even subconcussive events, those resulting in subclinical brain dysfunction without

26 the typical symptoms of concussion, may lead to long-term neurologic impairment ${ }^{11,12}$.

27 The immune response to TBI plays a fundamental role the development and progression

28 of subsequent neurodegenerative disease and represents a complex interplay between

29 the injured brain and the resident immune cells of the brain - the microglia ${ }^{13}$. The current

30 manuscript is focused on developing a cell-type-specific understanding of the microglial

31 response to injury over time. Here we highlight the major trends in gene expression in

32 response to TBI.

34 TBI triggers a robust pro-inflammatory response within the injured brain. The degree of

35 this initial pro-inflammatory response has significant value in predicting more long-term

36 outcomes after TBI ${ }^{14-16}$. Even after the acute inflammatory response has resolved,

37 several studies demonstrated residual long-lasting inflammation within the brain in both

38 animal models as well as in patients 17,18 . One of the main drivers of this continued

39 inflammation is the persistence of activated microglia-characterized by thickening and

40 retraction of their ramified processes, increased IL-1 and IL-6 with concomitant decreases

41 in IL-4 and IL-10, and increased expression of pro-oxidant genes with a reduction of

42 growth and antioxidant genes. A recent study showed an increased inflammatory profile

43 of microglia that persisted for up to 12 months after injury in a murine model of TBI ${ }^{19}$.

44 Furthermore, this continued inflammation is associated with lesion volume expansion and

45 loss of neurons in the hippocampus ${ }^{18-22}$. Even once the acute inflammatory process has

46 resolved and infiltrated monocyte-derived macrophages are no longer present within the 
47 injured brain, microglia have the potential to remain activated for years after the initial

48 insult ${ }^{19,23}$. A functional consequence of this constitutive activation after brain trauma is

49 an exaggerated neuroinflammatory response to otherwise benign secondary stimuli such

50 a subsequent subclinical head injury 10,24-27. This may be the mechanism by which

51 patients who have sustained a concussion are more susceptible to subsequent

52 concussions ${ }^{28-30}$. Nonetheless, the molecular mechanisms resulting in the constitutive

53 activation of microglia remain elusive ${ }^{21}$. Therefore, in the current study, we aimed to

54 study the transcriptional dynamics of constitutively activated microglia over the course of

55 brain injury.

57 The first step towards any cell-specific transcriptional analysis relies on obtaining 58 sufficient cells of interest with the highest purity. The historical standard for distinguishing 59 between microglia and infiltrating macrophages is immunohistochemistry. Although 60 immunohistochemistry is useful for assessing morphology, proliferation, and sites of

61 activation it has a number of drawbacks limiting its use ${ }^{31}$. Several investigative groups

62 have focused on this problem including column free magnetic separation and CD11b

63 immunomagnetic enrichment combined with the differential expression of CD45 with flow

64 cytometry ${ }^{31-33}$. However, CD45 expression has been reported to vary depending on the

65 pathologic condition; thus, reliable separation of microglia from peripheral myeloid cells

66 is impaired ${ }^{34,35}$. To overcome this issue, fluorescently marked myeloid cells, such as

$67 \mathrm{CX} 3 \mathrm{CR} 1^{+/ G F P} / \mathrm{CCR} 2^{+/ R F P}$, have been used. However, these mice are on mixed

68 backgrounds, which could greatly affect the immune response. Furthermore, the 69 presence of contaminated nonclassical monocytes could not be excluded ${ }^{36,37}$. Therefore, 
70 we use head-shielded bone marrow chimeric mice with CD45.1 cells in the circulation

71 and CD45.2 microglia in the brain allowing definitive and unambiguous differentiation

72 between microglia and infiltrating bone-marrow derived myeloid cells as previously

73 described by our laboratory ${ }^{38}$. To the best of our knowledge, no cell-type-specific study

74 has been conducted to specifically identify transcriptional changes in isolated populations

75 of microglia over the course of TBI. In the current study we demonstrate that TBI-

76 associated microglia adopt longitudinal changes in their transcriptional profile associated

77 with pathways linked to the development of motor, cognitive, and behavioral disorders.

79 Results

80 Global patterns of gene expression from isolated populations of

81 microglia over the time course of TBI

82 The neuroinflammatory response to $\mathrm{TBI}$ is central to both neuroprotection and

83 neurotoxicity after injury, but attempts to broadly target immune activation have been

84 unsuccessful in improving outcomes in TBI patients ${ }^{39-42}$. Because of this, there has been

85 a growing interest in investigating the microglial transcriptome after brain injury. Attempts

86 thus far have been plagued by the use of whole brain homogenates rather than individual

87 cell types as well as the use of microarray analyses restricted to a limited number of genes

88 in the inflammatory response ${ }^{43,44}$. Therefore, we combined our ability to discriminate and

89 sort microglia from infiltrating monocytes and macrophages with unbiased transcriptional

90 profiling (RNA-seq) on FACSorted microglia from 7, 30, and 90 days after TBI. We

91 defined 396 genes that change expression across the time course (see methods). We

92 clustered these differentially expressed genes into 4 main patterns of expression (Fig. 1). 
93 Clusters 2 represents genes involved in synaptic plasticity and is progressively

94 upregulated over the course of injury. Cluster 4 represents genes involved in the

95 regulation of membrane polarization with gene expression that is upregulated at 30 days

96 post-injury and then downregulated by 90 days post injury. Clusters 1 and 3 represent

97 genes involved in the host response to injury and lipid remodeling and both are

98 progressively downregulated over the course of injury. These data provide new insights

99 into the biology of microglial activation over the course of TBI.

100

101 Figure 1. Microglia from TBI mice show distinct time-dependent transcriptional

102 profiles. K-means clustering: Four clusters representing 392 genes are shown with

103 distinct time specific expression patterns. Averaged $\log _{2}(C P M+1) \geq 3$ of genes in each

104 group that had a log fold

105 change of at least 2 was

106 used to generate the heat

107 map. GO processes

108 associated with the genes

109 in each cluster was

110 determined using Gorilla

111 database.

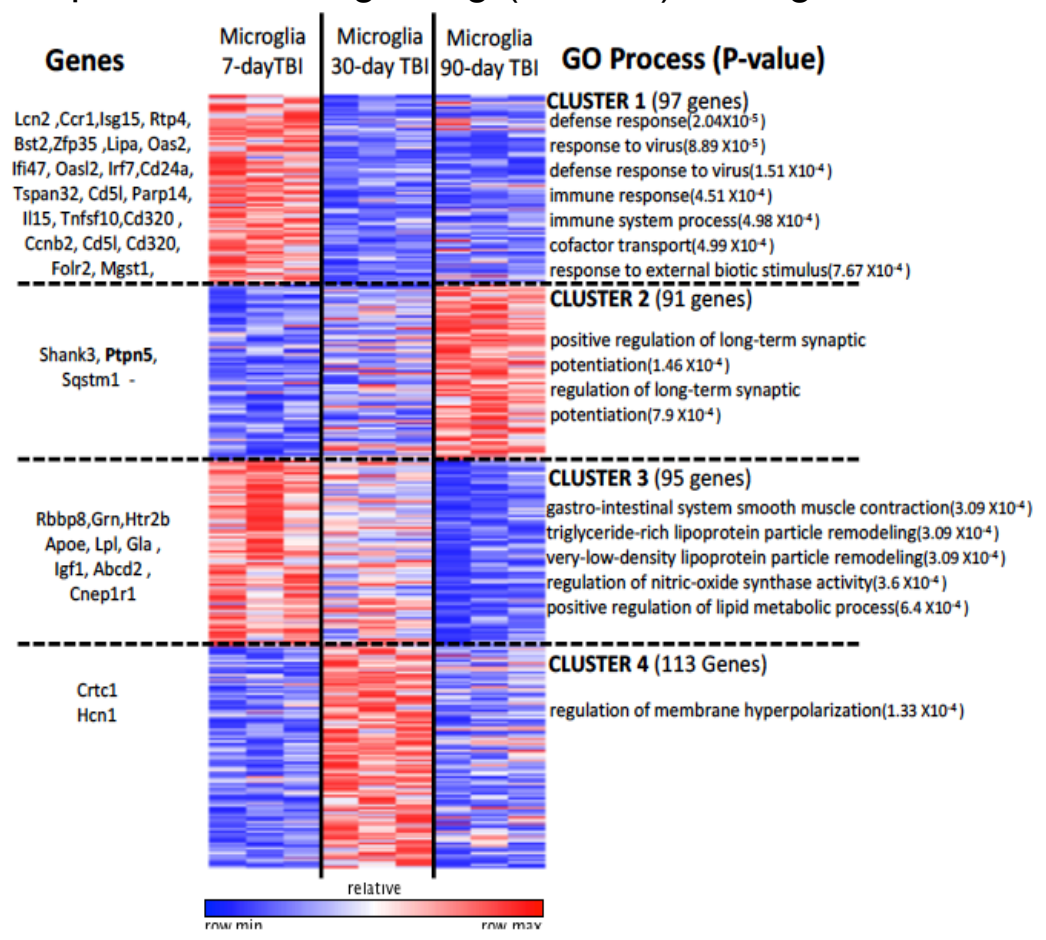

114 Pairwise comparison of microglia gene expression between time 
116 To further evaluate the gene expression over time in TBI-associated microglia, we

117 investigated differentially expressed genes between microglia across time points (Fig. 2).

118 We found 187 genes between days 7 and 30 that are positively differentiated and are

119 likely involved in channel transport activity, as well as defense response. Several genes

120 including genes involved in immune response and tissue repair such as Tnfsf10, Bst2,

121 Igf1, and Ccr1 were upregulated at the earlier time points. However, genes implicated in

122 neurodegenerative diseases such as Ptpn5, Shank3, and Sqstm1 were upregulated by

12390 days compared to 7 days post TBI. This expression profile is indicative of the chronic

124 inflammatory environment in the TBI brain following injury. To determine whether gene

125 expression trends at 30 days were predictive of 90 days post-TBI, we compared the fold

126 changes between 7 vs. 30 days post-TBI with those between 7 vs. 90 days post-TBI (Fig.

127 3). We find that these gene expression change were significantly correlated $(R=-0.537$,

$\left.128 \mathrm{p}=2.2 \times 10^{-16}\right)$. This indicates that genes which were upregulated at 90 days post-TBI had

129 already started to increase as early as 30 days post-injury. Conversely, those genes that

130 were downregulated at 30 days post-TBI remained downregulated or continued to fall

131 over time.

133 Figure 2: Differentially

134 expressed genes over the

135 time course of injury.

136 Volcano plots of differentially

137 expressed genes in microglia

138 between 7D and 30D post-TBI

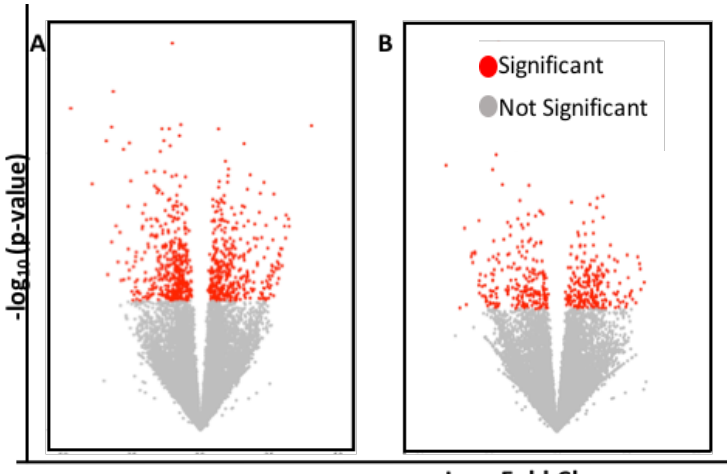

$\log _{2}$ Fold Change

$7 D$ vs $30 D$ post TBI

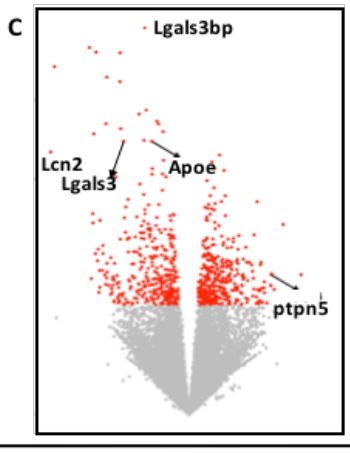

$7 D$ vs 900 post TBI 
(A), 30D and 90D post-TBI (B), and 7D and 90D post-TBI (C). N=3 in each group. Genes with a $p$ - value $<0.05$ are shown in red.

143 plot to examine the relationship between the genes that are up-regulated or down144 regulated in the microglia of mice at A) 7 vs 30 days post-TBI compared to 7 vs 90 days 145 post-TBI and at B) 30 vs 90 days post-TBI compared to 7 vs 90 days. A log2 fold change 146 of 1 is equal to a 2 -fold change.
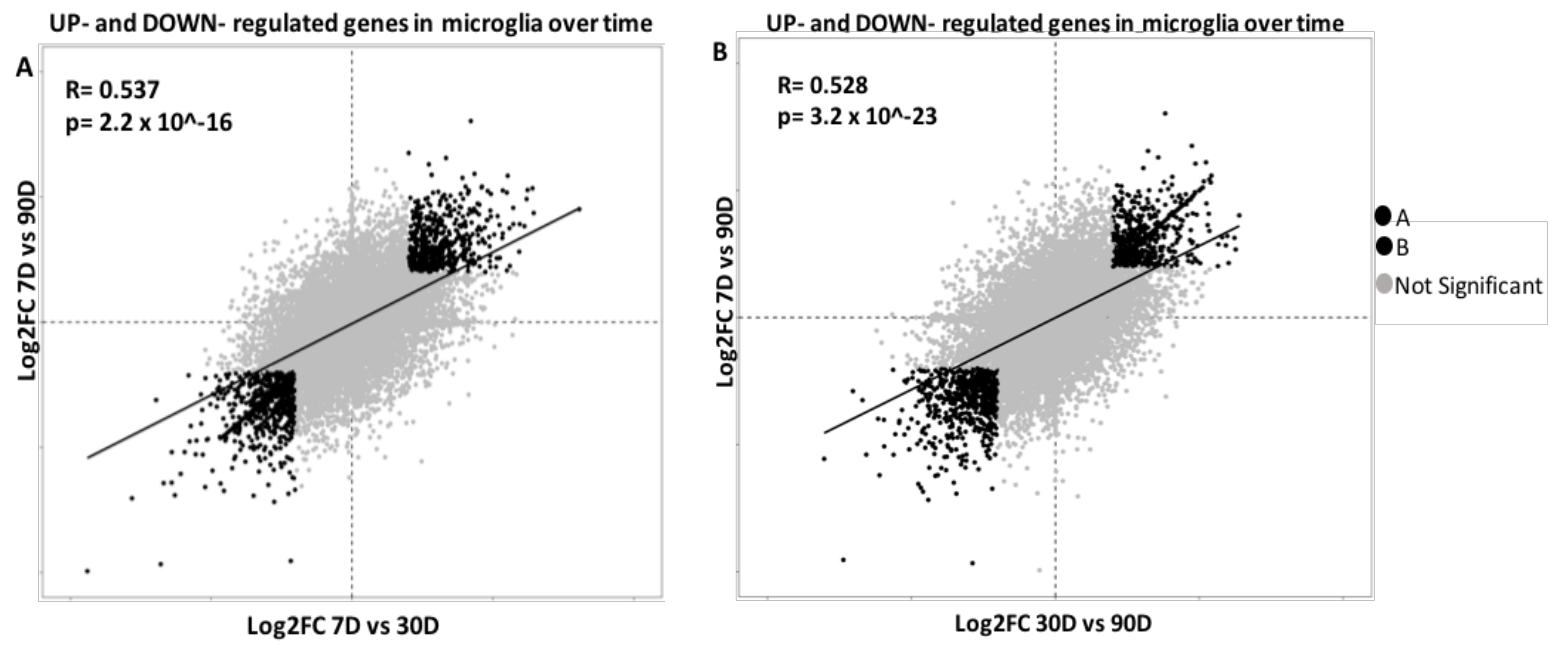

147

150 Microglia are essential to brain homeostasis but lose this homeostatic function in a 151 number of neurodegenerative disease processes. There has been considerable interest 152 in the Trem2-APOE pathway in the generation of a neurodegenerative microglial 153 phenotype in both Alzheimer's Disease (AD) and multiple sclerosis (MS). In fact, recent 154 data has identified the Trem2-APOE pathway as a pivotal regulator of microglial 
155 phenotype in both of these disease processes ${ }^{45}$. Therefore, we aimed to determine if the

156 Trem2-APOE pathway was a major regulator of microglial phenotype after TBI. However,

157 unlike other neurodegenerative processes, our data demonstrates no significant change

158 in TREM2 expression as well as a progressive decrease in APOE expression over the

159 course of TBI (Fig. 4). These seemingly contradictory results emphasize the need for

160 microglia-specific transcriptional studies in the setting TBI.

161

162 Figure 4. Microglial Trem2 and APOE expression over the course of TBI. Microglial

163 Trem2 did not significantly change

164 over the course of brain injury.

165 APOE expression progressively

166 decreased over time following TBI.

167 Ordinary one way ANOVA with

168 Bonferroni's multiple comparison

169 test.

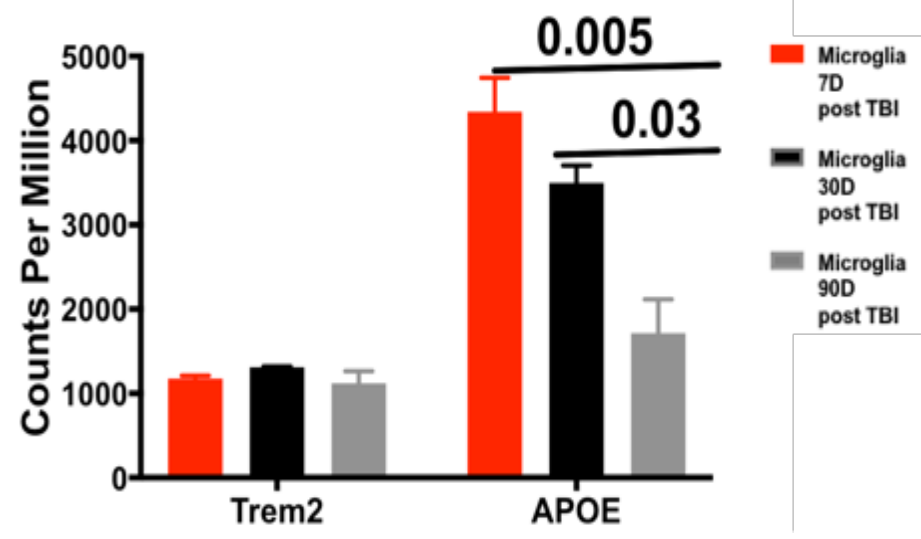

171 Microglial STEP expression is progressively upregulated over the 172 course of brain injury.

173 While our data failed to demonstrate a common pathway with Alzheimer's disease and

174 multiple sclerosis through Trem2-APOE, a closer examination of Cluster 2 (Fig. 1) revealed

175 a number of upregulated genes associated with long-term synaptic potentiation, including PTPN5

176 also known as STEP (STriatial-Enriched protein tyrosine Phosphatase). STEP is a brain-specific

177 phosphatase and is highly expressed within the cortex, hippocampus, and amygdala ${ }^{46,47}$. Our 
data show that expression of STEP is progressively increased in microglia over time following TBI

(Fig. 5). STEP is critical in the long-term depression, or weakening, of synaptic efficacy between neurons-a process fundamental to learning, memory, and cognition ${ }^{48,49}$. When STEP activity is elevated, several substrates are inactivated resulting in the internalization of NMDA/AMPA 182 glutamate receptors ${ }^{50}$. This disrupts synaptic function and contributes to cognitive deficits ${ }^{49,51}$.

183 In fact, elevated STEP is associated with the pathophysiology of Alzheimer's disease, 184 schizophrenia, and ischemic brain injury in both human cortex and mouse models ${ }^{52-57}$. These 185 data suggest that STEP may be one of the common molecular pathways connecting TBI with 186 other known neurodegenerative disorders.

Figure 5. Microglial STEP expression is

progressively upregulated over the course

190 of brain injury. STEP expression from

191 FACSorted microglia progressively increased

192 from 7 days post-TBI to 90 days post-TBI;

$193 p=0.01$, Ordinary one way ANOVA with

194 Bonferroni's multiple comparison test.

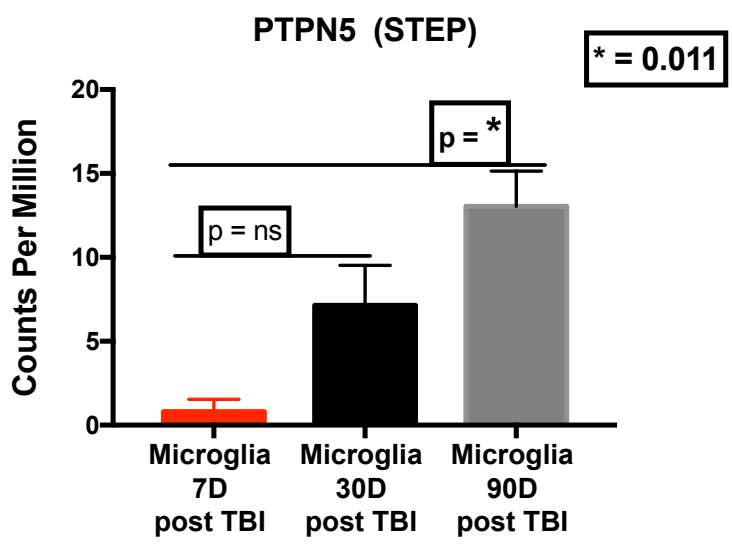

\section{Discussion}

197 Microglia are the resident innate immune cells of the CNS. They are ontologically distinct 198 from peripheral bone marrow-derived monocytes and macrophages, arising from the yolk sac as opposed to the developing liver in the embryo ${ }^{58}$. In fact, microglia rely on a 200 distinctive set of transcription factors during development resulting in a lineage of tissue 201 macrophages (microglia) derived from the yolk sac that are genetically distinct from bone 
202 marrow-derived macrophages ${ }^{37,59-62}$. Additionally, microglia are self-renewing suggesting

203 that monocyte-derived macrophages do not contribute to the maintenance of the mature

204 microglia pool 63,64. Distinct developmental origin and renewal mechanisms may suggest

205 that microglia possess discrete functions in pathological processes ${ }^{58}$. Despite this, the

206 cellular mechanisms by which microglia promote or attenuate the progression of injury

207 are largely unknown ${ }^{65}$. Our Data are the first we know of to use unbiased transcriptional

208 profiling of isolated populations of microglia to define the genes/pathways/signatures

209 involved in the generation of TBI-associated microglia over the course of injury.

211 To adequately capture the heterogeneity and complexity of microglia at different stages

212 of injury, a comprehensive, genome-wide sampling of individual cell types is required ${ }^{37,66}$.

213 A cell-specific delineation of innate immune function based on transcriptional profiling in

214 TBI has yet to be undertaken. Therefore, we combined our ability to discriminate and sort

215 microglia from infiltrating monocytes and monocyte-derived macrophages with unbiased

216 transcriptional profiling (RNA-seq) on FACSorted microglia. Our analysis identified 4

217 sequentially upregulated or downregulated gene clusters involved in processes such as

218 the host defense response, synaptic potentiation, lipid remodeling, and membrane

219 polarization each providing new insights into the biology of microglial activation over the

220 course of TBI (Fig. 1). While there has recently been considerable recent interest in the

221 Trem2-APOE pathway in the generation of a neurodegenerative microglial phenotype in

222 Alzheimer's Disease and multiple sclerosis ${ }^{45}$, our data demonstrates no significant

223 change in Trem2 expression as well as a progressive decrease in APOE expression over

224 the course of TBI (Fig. 4). However, an examination of cluster II revealed a number of 
225 genes associated with long-term synaptic potentiation, including PTPN5 also known as

226 STEP (STriatial-Enriched protein tyrosine Phosphatase). STEP is a brain-specific

227 phosphatase that is highly expressed within the striatum, cortex, hippocampus, and

228 amygdala 46,47 . STEP is critical in the long-term depression, or weakening, of synaptic

229 efficacy between neurons-a process fundamental to learning, memory, and cognition

23048,49 . Elevated STEP is associated with the pathophysiology of Alzheimer's disease (AD),

231 schizophrenia, and ischemic brain injury in both human cortex and mouse models ${ }^{52-57 .}$

232 In fact, genetic knockout of STEP reverses many of the cognitive and behavioral deficits

233 in AD models ${ }^{53,67}$. To the best of our knowledge, STEP expression has never been

234 studied within the context of TBI. Our RNA-seq analysis demonstrates a 13-fold increase

235 in microglial expression of STEP over the course of injury (Fig. 5). Previous studies have

236 shown that STEP affects neuronal communication by opposing synaptic strengthening.

237 High levels of STEP are believed to disrupt synaptic function and to contribute to learning

238 deficits in neurodegenerative disease ${ }^{52,68}$. When STEP activity is elevated, several

239 substrates are inactivated resulting in the internalization of NMDA/AMPA glutamate

240 receptors ${ }^{50}$. This disrupts synaptic function and contributes to cognitive deficits ${ }^{49,51}$. In

241 other words, STEP activation modulates learning and memory by removing glutamate

242 receptors from synaptic membranes. This important discovery suggests that TBI may

243 share a common molecular pathway with several other cognitive disorders previously

244 regarded as distinct. These data are remarkable in demonstrating the power of

245 longitudinal transcriptional profiling, which provides important biologic insights into the

246 state of microglial processes even in the complex and dynamic model of traumatic brain 
247 injury. Furthermore, these data strongly implicate longitudinal changes in microglial gene expression in the development of long-term neurocognitive changes.

250 In conclusion, our data demonstrate that TBI-associated microglia adopt longitudinal

251 transcriptional changes consistent with long-term depression of synaptic strength. The

252 contribution of altered microglial gene expression to the pathogenesis of TBI has not been

253 previously investigated. Our data suggest that TBI-associated microglia may play a

254 previously unknown role in the weakening of synaptic efficacy between neurons after

255 brain injury. As a result, learning, memory, and cognitive performance may all be affected

256 leading to the resultant long-term neurocognitive impairments seen after TBI. Moving

257 forward it will be important to study larger cohorts of brain-injured mice during both the

258 acute and chronic phase of TBI. Furthermore, it has been shown that microglia display

259 different transcriptional identities depending on the brain region in which they reside as

260 well as their age ${ }^{69}$. This will necessitate side-by-side comparison with age-matched naïve

261 control mice to account for transcriptional changes associated with aging. Additionally, a

262 single-cell RNA-seq approach may be required to account for inherent microglial

263 heterogeneity at the site of injury. This could allow for the identification of novel microglial

264 subpopulations within and surrounding the site of injury. Regardless of the techniques

265 used, once the molecular mechanisms underlying the transcriptional changes in microglia

266 after injury are further delineated, targeting the microglial response after TBI may soon

267 represent a target for future therapeutic intervention. 


\section{Mice}

271 All procedures were approved by the Northwestern University Institutional Animal Care

272 and Use committee and all experiments were carried out in accordance with the ARRIVE

273 guidelines on the reporting of in vivo experiments. Two mouse strains were used, they

274 are C57BL/6 and B6.SJL-Ptprc ${ }^{a} P e p c^{b} /$ BoyJ (CD45.1). All mice were purchased from the

275 Jackson Laboratory and housed at a barrier facility at the Center for Comparative

276 Medicine at Northwestern University (Chicago, IL, USA). Sixteen-week-old mice were

277 used for all experiments.

\section{Shielded Bone Marrow Chimeras}

279 Bone marrow was aseptically harvested from tibias and femurs, from 8-week-old

280 B6.CD45.1 donor mice, erythrocytes were lysed and the cells were counted using a

281 Countess automated cell counter. 8 weeks old B6.CD45. 2 mice received a single 1000-

282 cGy y-irradiation dose using a Cs-137-based Gammacell 40 irradiator. The mice heads

283 were shielded with a lead bar so as to deliver the irradiation to the body only. 6 hours

284 after shielded irradiation, busulfan $(30 \mathrm{mg} / \mathrm{kg})$ was administered to completely ablate the

285 bone marrow of the recipient mice. Donor bone marrow (CD45.1) was transplanted 12

286 hours after busulfan ablation. Shielded bone marrow chimeras were maintained on

287 antibiotics trimethoprim/sulfamethoxazole (40 mg/5 mg, respectively). Eight weeks after

288 irradiation, $95 \%$ of the circulating monocytes were of donor origin (Fig. 6) ${ }^{38}$. 
Figure 6. Microglia from head-shielded bone marrow chimeric mice are host origin.

291 Brains isolated from chimeric mice post TBI were analyzed by flow cytometry. CD45.1 $1^{\text {neg }}$

292 'B, T, NK cells, Eosinophils' acted as the gating control for CD45.2 hi, showing that resident

293 microglia $\left(\mathrm{CD} 45^{\mathrm{lo}}\right)$

294 can be

295 unambiguously

296 differentiated from

297 infiltrating monocyte-

1000-cGy $\gamma$-irradiation Transfer B6.CD45.1

with brain shielding donor bone marrow

298 derived macrophages

$299 \quad\left(C D 45.1^{+}\right) . \quad$ Arrows

300 indicated the

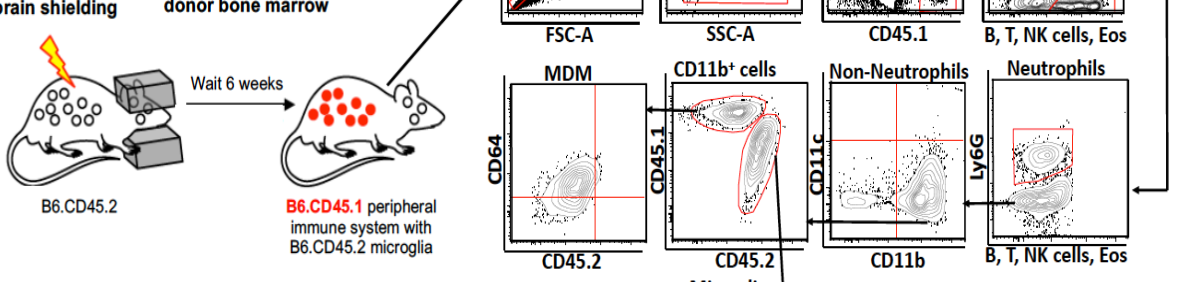

301 directionality of gating.

\section{Controlled cortical impact}

304 Controlled cortical impact was induced as previously described by our laboratory ${ }^{38}$. In

305 brief, mice were anesthetized with $100 \mathrm{mg} / \mathrm{kg}$ Ketamine and $10 \mathrm{mg} / \mathrm{kg}$ Xylazine via 306 intraperitoneal injection. A $1 \mathrm{~cm}$ scalp incision was performed and a $5 \mathrm{~mm}$ craniectomy

307 was performed $2 \mathrm{~mm}$ left of the sagittal suture and $2 \mathrm{~mm}$ rostral to the coronal suture.

308 The dura was left intact. Mice were then placed in a stereotaxic operating frame and the

309 impactor (Leica Biosystems Inc., Buffalo Grove, IL) was maneuvered into position. A

310 controlled cortical impact was then applied with a $3 \mathrm{~mm}$ impacting tip at a velocity of $2.5 \mathrm{~m} / \mathrm{s}$

311 and an impacting depth of $2 \mathrm{~mm}$ with the dwell time set at $0.1 \mathrm{~s}$. Immediately following 312 injury all animals had their scalps sealed with VetBond (3M). All animals received post 
313 injury analgesia with Buprenorphine SR via subcutaneous injection and were allowed to

314 recover in separate cages over a warming pad. Mice were euthanized at 7, 30, and 90

315 days post TBI and brains were harvested.

316

317 Tissue preparation and fluorescence activated cell sorting

318 Immediately following euthanasia, mice were transcardially perfused first with ice cold PBS.

319 The brains were then excised and place in ice cold HBSS until time to process. The brains

320 were weighed, cut into pieces and placed into C-tubes containing digestion buffer (2.5

$321 \mathrm{mg} / \mathrm{mL}$ Liberase TL (Roche, Basel, Switzerland), and $1 \mathrm{mg} / \mathrm{mL}$ of DNase I in HBSS). The

322 C-tubes were placed on a MACS dissociator (Miltenyi Biotec) and run on the M_Brain_3

323 protocol, after which they were placed in an incubator for 30 minutes at $37^{\circ} \mathrm{C}$ with shaking

324 at $200 \mathrm{rpm}$. After incubation, the C-tubes were placed back on the MACS dissociator and

325 run on the same protocol as before. The cells released were then passed through a $40 \mu \mathrm{m}$

326 nylon mesh and washed with $100 \mathrm{ml}$ of wash buffer (1\% BSA in HBSS) per brain sample.

327 Microglia and infiltrating cells were isolated using a 30/70 percoll gradient (Percoll Plus, GE

328 Healthcare). The cells collected from the interphase of the gradient were washed with

329 HBSS and counted using Countess automated cell counter (Invitrogen); dead cells were

330 discriminated using trypan blue. Cells were stained with live/dead Aqua (Invitrogen) viability

331 dye, incubated with Fc-Block (BD Bioscience) and stained with fluorochrome-conjugated

332 antibodies (Table 1). Data were acquired on a BD FACSAria cell sorter (BD Biosciences,

333 San Jose, CA), and microglia were sorted for further analyses. "Fluorescence minus one"

334 controls were used when necessary to set up gates. Pelleted sorted cells were immediately

335 lysed in extraction buffer from a PicoPure RNA isolation kit (Arcturus Bioscience), and 
336 lysates were stored at $-80^{\circ} \mathrm{C}$ until RNA was extracted. Analysis of the flow cytometric data 337 was performed using Flowjo software (TreeStar, Ashland, OR).

\begin{tabular}{|l|l|l|}
\hline Antibody & Fluorochrome & Company and Clone \\
\hline CD45.1 & FITC & A-20 / BD Bioscience \\
\hline CD45.2 & BV421 & 104 / Biolegend \\
\hline CD64 & APC & X54-5/7.1/ BD Bioscience \\
\hline CD11b & APC- Cy7 & M1/70 / BD Biosciences \\
\hline CD11c & PE-Cy7 & HL3 / BD Bioscience \\
\hline Ly6G & Alexa Flour 700 & 1 A8 / BD Bioscience \\
\hline MHC II & Percp- Cy 5.5 & M5/114.15.2 / Biolegend \\
\hline Siglec H & PE & $551 /$ Biolegend \\
\hline B220 & PECF594 & RA3-6B2 / BD Bioscience \\
\hline Siglec F & PECF594 & E50-2440 / BD Bioscience \\
\hline CD4 & PECF594 & RM4-5/ BD Bioscience \\
\hline CD8 & PECF594 & $53-6.7$ / BD Bioscience \\
\hline NK1.1 & PECF594 & PK136/ BD Bioscience \\
\hline Viability & e-bioscience Fixable & Invitrogen \\
& Viability Dye eFluor 506 & \\
\hline
\end{tabular}

Table 1. List of antibody conjugated fluorochromes used to differentiate microglia from infiltrating leukocytes.

\section{RNA sequencing}

343 RNA from the FACSorted microglia of brain-injured mice were extracted using a PicoPure

344 RNA isolation kit according to manufacturer's instructions. Sample quality control,

345 processing, and library preparation were performed by the Northwestern University Next

346 Generation Sequencing Core (NUSeq). RNA quality and quantity were measured using

347 Agilent High Sensitivity RNA ScreenTape System (Agilent Technologies). RNA

348 sequencing (RNA-seq) libraries were prepared from 3ng of total RNA using the QuantSeq

349 3' biased mRNA-seq Library Prep Kit for Illumina (Lexogen). DNA libraries were 
350 sequenced on an Illumina NextSeq 500 instrument with a target read depth of $\sim 20$ million

351 reads per sample.

\section{RNA-seq analysis}

354 Raw sequencing files were first de-multiplexed using bcl2fastq. The resulting fastq files

355 were trimmed of low-quality reads and bases, polyA tails, and adaptors using bbduk

356 (http://jgi.doe.gov/data-and-tools/bb-tools/). The trimmed fastq files were aligned to the

357 mouse reference genome (mm10, Genome Reference Consortium GRCm38) using the

358 STAR (Spliced Transcripts Alignment to a Reference) algorithm ${ }^{70}$. HTSeq was run on

359 the resulting BAM files to provide raw gene counts. Raw gene counts for each sample

360 were merged into a single gene expression table and normalized for read depth using

361 counts per million (CPM). The three highest quality samples, based on RNA quality and

362 library quality from each experimental group were included for subsequent analyses. For

363 the RNA-seq analysis, we focused on the expressed genes which were defined as average

$364 \log \mathrm{CPM}\left(\log _{2}(\mathrm{CPM}+1)\right)$ expression > 4 in each experimental group. For visualization, GENE-E

365 (https://software.broadinstitute.org/GENE-E/) was used to perform K-means clustering

$366(\mathrm{~K}=4)$ on differentially expressed genes across all time points as defined by ANOVA test

$367(p<0.05)$ across any two groups shown in the heatmap. Gene Ontology associations and

368 the related p-values were determined by GO analysis (by GOrilla- [Gene Ontology

369 enRIchment anaLysis and visuaLizAtion tool]).

$370 \quad 71$. Pairwise differential genes between time points were determined using DEseq2.

371 Volcano plots were generated using the log2 fold change of normalized gene counts

372 between microglia at different time points on the x-axis and corresponding p-values (- 
$373 \log 10)$ from DEseq2 on the $y$-axis. Plots were generated using the ggplot2 package from

374 R Studio software.

375

376 Data Availability

377 The data that support the findings of this study are available from the corresponding

378 author on request. RNA sequencing data is available through the NCBI Sequence

379 Read Archive (SRA accession number: SRP160379).

\section{References}

1 Faul, M. Traumatic Brain Injury in the United States: Emergency Department Visits, Hospitalizations and Deaths 2002-2006. (Centers for Disease Control and Prevention,

2 Roozenbeek, B., Maas, A. I. \& Menon, D. K. Changing patterns in the epidemiology of National Center for Injury Prevention and Control, Atlanta (GA), 2010).

401

402 traumatic brain injury. Nat Rev Neurol 9, 231-236, doi:10.1038/nrneurol.2013.22 (2013).

3 Corso, P., Finkelstein, E., Miller, T., Fiebelkorn, I. \& Zaloshnja, E. Incidence and lifetime costs of injuries in the United States. Injury prevention : journal of the International Society for Child and Adolescent Injury Prevention 12, 212-218, doi:10.1136/ip.2005.010983 (2006).

4 Pearson, W. S., Sugerman, D. E., McGuire, L. C. \& Coronado, V. G. Emergency department visits for traumatic brain injury in older adults in the United States: 2006-08. The western journal of emergency medicine 13, 289-293, doi:10.5811/westjem.2012.3.11559 (2012).

5 Whitlock, J. A., Jr. \& Hamilton, B. B. Functional outcome after rehabilitation for severe traumatic brain injury. Archives of physical medicine and rehabilitation 76, 1103-1112 (1995).

6 Schwarzbold, M. et al. Psychiatric disorders and traumatic brain injury. Neuropsychiatric disease and treatment 4, 797-816 (2008).

7 Whelan-Goodinson, R., Ponsford, J., Johnston, L. \& Grant, F. Psychiatric disorders following traumatic brain injury: their nature and frequency. The Journal of head trauma rehabilitation 24, 324-332, doi:10.1097/HTR.0b013e3181a712aa (2009). 
4079 Martin, L. A., Neighbors, H. W. \& Griffith, D. M. The experience of symptoms of 408 depression in men vs women: analysis of the National Comorbidity Survey Replication. JAMA psychiatry 70, 1100-1106, doi:10.1001/jamapsychiatry.2013.1985 (2013). Makinde, H. M., Just, T. B., Cuda, C. M., Perlman, H. \& Schwulst, S. J. The Role of Microglia in the Etiology and Evolution of Chronic Traumatic Encephalopathy. Shock 48,

11 Belanger, H. G., Vanderploeg, R. D. \& McAllister, T. Subconcussive Blows to the Head: A Formative Review of Short-term Clinical Outcomes. J Head Trauma Rehabil 31, 159-166, doi:10.1097/HTR.0000000000000138 (2016).

Carman, A. J. et al. Expert consensus document: Mind the gaps-advancing research into short-term and long-term neuropsychological outcomes of youth sports-related concussions. Nat Rev Neurol 11, 230-244, doi:10.1038/nrneurol.2015.30 (2015). Hernandez-Ontiveros, D. G. et al. Microglia activation as a biomarker for traumatic brain injury. Front Neurol 4, 30, doi:10.3389/fneur.2013.00030 (2013). neuroinflammation, polytrauma, and outcome. Brain Behav Immun 45, 253-262, doi:10.1016/j.bbi.2014.12.021 (2015). interleukin-6 levels correlate with improved outcome after traumatic brain injury. Brain 127, 315-320, doi:10.1093/brain/awh039 (2004). Injury. Front Neurol 8, 351, doi:10.3389/fneur.2017.00351 (2017). Kumar, R. G., Boles, J. A. \& Wagner, A. K. Chronic Inflammation After Severe Traumatic Brain Injury: Characterization and Associations With Outcome at 6 and 12 Months Postinjury. The Journal of head trauma rehabilitation 30, 369-381, doi:10.1097/HTR.0000000000000067 (2015).

18 Smith, D. H., Johnson, V. E. \& Stewart, W. Chronic neuropathologies of single and repetitive TBI: substrates of dementia? Nature reviews. Neurology 9, 211-221, doi:10.1038/nrneurol.2013.29 (2013). neurodegeneration after experimental brain trauma: association with chronic microglial activation. J Neuropathol Exp Neurol 73, 14-29, doi:10.1097/NEN.0000000000000021 (2014). clinical consequences, and therapeutic opportunities. Neuropsychiatric disease and treatment 11, 97-106, doi:10.2147/NDT.S65815 (2015).

21 Perry, V. H. \& Holmes, C. Microglial priming in neurodegenerative disease. Nat Rev Neurol 10, 217-224, doi:10.1038/nrneurol.2014.38 (2014).

22 Henry, C. J., Huang, Y., Wynne, A. M. \& Godbout, J. P. Peripheral lipopolysaccharide (LPS) challenge promotes microglial hyperactivity in aged mice that is associated with exaggerated induction of both pro-inflammatory IL-1beta and anti-inflammatory IL-10 cytokines. Brain Behav Immun 23, 309-317, doi:10.1016/j.bbi.2008.09.002 (2009). a single traumatic brain injury. Brain 136, 28-42, doi:10.1093/brain/aws322 (2013). 
45124 Field, R., Campion, S., Warren, C., Murray, C. \& Cunningham, C. Systemic challenge with the TLR3 agonist poly I:C induces amplified IFNalpha/beta and IL-1beta responses in the diseased brain and exacerbates chronic neurodegeneration. Brain Behav Immun 24, 996-1007, doi:10.1016/j.bbi.2010.04.004 (2010).

25 Ohmoto, Y. et al. Variation in the immune response to adenoviral vectors in the brain: influence of mouse strain, environmental conditions and priming. Gene Ther 6, 471-481, doi:10.1038/sj.gt.3300851 (1999).

26 McColl, B. W., Rothwell, N. J. \& Allan, S. M. Systemic inflammatory stimulus potentiates the acute phase and CXC chemokine responses to experimental stroke and exacerbates

27 Schroder, K., Sweet, M. J. \& Hume, D. A. Signal integration between IFNgamma and TLR brain damage via interleukin-1- and neutrophil-dependent mechanisms. The Journal of neuroscience : the official journal of the Society for Neuroscience 27, 4403-4412, signalling pathways in macrophages. Immunobiology 211, 511-524, doi:10.1016/j.imbio.2006.05.007 (2006). Muccigrosso, M. M. et al. Cognitive deficits develop 1month after diffuse brain injury and are exaggerated by microglia-associated reactivity to peripheral immune challenge. Brain Behav Immun 54, 95-109, doi:10.1016/j.bbi.2016.01.009 (2016). Weil, Z. M., Gaier, K. R. \& Karelina, K. Injury timing alters metabolic, inflammatory and functional outcomes following repeated mild traumatic brain injury. Neurobiol Dis 70, 108-116, doi:10.1016/j.nbd.2014.06.016 (2014). Foris, L. A. \& Donnally, I. C. in StatPearls (2017). Nikodemova, M. \& Watters, J. J. Efficient isolation of live microglia with preserved phenotypes from adult mouse brain. Journal of neuroinflammation 9, 147, doi:10.1186/1742-2094-9-147 (2012). flow cytometric characterization of mouse microglia. Journal of neuroscience methods 219, 176-182, doi:10.1016/j.jneumeth.2013.07.017 (2013). Gordon, R. et al. A simple magnetic separation method for high-yield isolation of pure primary microglia. Journal of neuroscience methods 194, 287-296, doi:10.1016/j.jneumeth.2010.11.001 (2011). injury in the female rat. Journal of neuroinflammation 11, 82, doi:10.1186/1742-209411-82 (2014).

35 Trahanas, D. M., Cuda, C. M., Perlman, H. \& Schwulst, S. J. Differential Activation of Infiltrating Monocyte-Derived Cells After Mild and Severe Traumatic Brain Injury. Shock 43, 255-260, doi:10.1097/SHK.0000000000000291 (2015).

36 Noristani, H. N. et al. RNA-Seq Analysis of Microglia Reveals Time-Dependent Activation of Specific Genetic Programs following Spinal Cord Injury. Front Mol Neurosci 10, 90, doi:10.3389/fnmol.2017.00090 (2017).

37 Matcovitch-Natan, O. et al. Microglia development follows a stepwise program to regulate brain homeostasis. Science 353, aad8670, doi:10.1126/science.aad8670 (2016).

38 Makinde, H. M., Cuda, C. M., Just, T. B., Perlman, H. R. \& Schwulst, S. J. Nonclassical Monocytes Mediate Secondary Injury, Neurocognitive Outcome, and Neutrophil 
Infiltration after Traumatic Brain Injury. J Immunol 199, 3583-3591, doi:10.4049/jimmunol.1700896 (2017).

39 Schwarzmaier, S. M. \& Plesnila, N. Contributions of the immune system to the pathophysiology of traumatic brain injury - evidence by intravital microscopy. Frontiers in cellular neuroscience 8, 358, doi:10.3389/fncel.2014.00358 (2014).

40 de Rivero Vaccari, J. P., Dietrich, W. D. \& Keane, R. W. Activation and regulation of cellular inflammasomes: gaps in our knowledge for central nervous system injury. Journal of cerebral blood flow and metabolism : official journal of the International Society of Cerebral Blood Flow and Metabolism 34, 369-375, doi:10.1038/jcbfm.2013.227 (2014).

41 Edwards, P. et al. Final results of MRC CRASH, a randomised placebo-controlled trial of intravenous corticosteroid in adults with head injury-outcomes at 6 months. Lancet 365, 1957-1959, doi:10.1016/S0140-6736(05)66552-X (2005).

42 Roberts, I. et al. Effect of intravenous corticosteroids on death within 14 days in 10008 adults with clinically significant head injury (MRC CRASH trial): randomised placebocontrolled trial. Lancet 364, 1321-1328, doi:10.1016/S0140-6736(04)17188-2 (2004).

43 Wei, H. H. et al. NNZ-2566 treatment inhibits neuroinflammation and pro-inflammatory cytokine expression induced by experimental penetrating ballistic-like brain injury in rats. Journal of neuroinflammation 6, 19, doi:10.1186/1742-2094-6-19 (2009).

44 Cao, T., Thomas, T. C., Ziebell, J. M., Pauly, J. R. \& Lifshitz, J. Morphological and genetic activation of microglia after diffuse traumatic brain injury in the rat. Neuroscience 225, 65-75, doi:10.1016/j.neuroscience.2012.08.058 (2012).

45 Krasemann, S. et al. The TREM2-APOE Pathway Drives the Transcriptional Phenotype of Dysfunctional Microglia in Neurodegenerative Diseases. Immunity 47, 566-581 e569, doi:10.1016/j.immuni.2017.08.008 (2017).

46 Boulanger, L. M. et al. Cellular and molecular characterization of a brain-enriched protein tyrosine phosphatase. The Journal of neuroscience : the official journal of the Society for Neuroscience 15, 1532-1544 (1995).

47 Lombroso, P. J., Naegele, J. R., Sharma, E. \& Lerner, M. A protein tyrosine phosphatase expressed within dopaminoceptive neurons of the basal ganglia and related structures. The Journal of neuroscience : the official journal of the Society for Neuroscience 13, 3064-3074 (1993).

48 Bliss, T. V. \& Collingridge, G. L. A synaptic model of memory: long-term potentiation in the hippocampus. Nature 361, 31-39, doi:10.1038/361031a0 (1993).

49 Silva, A. J. Molecular and cellular cognitive studies of the role of synaptic plasticity in memory. J Neurobiol 54, 224-237, doi:10.1002/neu.10169 (2003).

50 Braithwaite, S. P. et al. Regulation of NMDA receptor trafficking and function by striatalenriched tyrosine phosphatase (STEP). The European journal of neuroscience 23, 28472856, doi:10.1111/j.1460-9568.2006.04837.x (2006).

51 Chin, J. et al. Fyn kinase induces synaptic and cognitive impairments in a transgenic mouse model of Alzheimer's disease. The Journal of neuroscience : the official journal of the Society for Neuroscience 25, 9694-9703, doi:10.1523/JNEUROSCI.2980-05.2005 (2005). 
$53852 \quad$ Kurup, P. et al. Abeta-mediated NMDA receptor endocytosis in Alzheimer's disease involves ubiquitination of the tyrosine phosphatase STEP61. The Journal of neuroscience : the official journal of the Society for Neuroscience 30, 5948-5957, doi:10.1523/JNEUROSCI.0157-10.2010 (2010). reverses cognitive and cellular deficits in an Alzheimer's disease mouse model. Proceedings of the National Academy of Sciences of the United States of America 107, 19014-19019, doi:10.1073/pnas.1013543107 (2010).

54 Zhang, Y. et al. Reduced levels of the tyrosine phosphatase STEP block beta amyloidmediated GluA1/GluA2 receptor internalization. Journal of neurochemistry 119, 664672, doi:10.1111/j.1471-4159.2011.07450.x (2011).

55 Gold, J. M. \& Harvey, P. D. Cognitive deficits in schizophrenia. Psychiatr Clin North Am 16, 295-312 (1993). difficulties in patients with Huntington's disease. Nurs Stand 20, 41-44, doi:10.7748/ns2006.05.20.35.41.c4146 (2006). Anderson, C. A. \& Arciniegas, D. B. Cognitive sequelae of hypoxic-ischemic brain injury: a review. NeuroRehabilitation 26, 47-63, doi:10.3233/NRE-2010-0535 (2010). primitive macrophages. Science 330, 841-845, doi:10.1126/science.1194637 (2010). Schulz, C. et al. A lineage of myeloid cells independent of Myb and hematopoietic stem cells. Science 336, 86-90, doi:10.1126/science.1219179 (2012). "resident" myeloid cells: the case of the microglia. Glia 61, 112-120, doi:10.1002/glia.22393 (2013). functional signature in microglia. Nature neuroscience 17, 131-143, doi:10.1038/nn.3599 (2014). microenvironment. Cell 159, 1312-1326, doi:10.1016/j.cell.2014.11.018 (2014). sustain CNS microglia maintenance and function throughout adult life. Nature neuroscience 10, 1538-1543, doi:10.1038/nn2014 (2007). Aguzzi, A., Barres, B. A. \& Bennett, M. L. Microglia: scapegoat, saboteur, or something else? Science 339, 156-161, doi:10.1126/science.1227901 (2013).

65 Ajami, B., Bennett, J. L., Krieger, C., McNagny, K. M. \& Rossi, F. M. Infiltrating monocytes trigger EAE progression, but do not contribute to the resident microglia pool. Nature neuroscience 14, 1142-1149, doi:10.1038/nn.2887 (2011).

66 Zeisel, A. et al. Brain structure. Cell types in the mouse cortex and hippocampus revealed by single-cell RNA-seq. Science 347, 1138-1142, doi:10.1126/science.aaa1934 (2015).

67 Goebel-Goody, S. M. et al. Genetic manipulation of STEP reverses behavioral abnormalities in a fragile X syndrome mouse model. Genes Brain Behav 11, 586-600, doi:10.1111/j.1601-183X.2012.00781.x (2012). 
58268 Poddar, R., Deb, I., Mukherjee, S. \& Paul, S. NR2B-NMDA receptor mediated modulation 583 of the tyrosine phosphatase STEP regulates glutamate induced neuronal cell death.

$584 \quad$ Journal of neurochemistry 115, 1350-1362, doi:10.1111/j.1471-4159.2010.07035.x

585 (2010).

$58669 \quad$ Grabert, K. et al. Microglial brain region-dependent diversity and selective regional

587 sensitivities to aging. Nat Neurosci 19, 504-516, doi:10.1038/nn.4222 (2016).

58870 Dobin, A. et al. STAR: ultrafast universal RNA-seq aligner. Bioinformatics 29, 15-21,

589 doi:10.1093/bioinformatics/bts635 (2013).

59071 Eden, E., Navon, R., Steinfeld, I., Lipson, D. \& Yakhini, Z. GOrilla: a tool for discovery and 591 visualization of enriched GO terms in ranked gene lists. BMC Bioinformatics 10, 48, doi:10.1186/1471-2105-10-48 (2009).

\section{Author Contributions}

S.S and H.M wrote the main manuscript text and prepared all figures. H.M, T.J

596 and S.S carried out the experiments. H.M and D.W performed the computational analysis

597 of the sequencing data. 